RC567

B38D
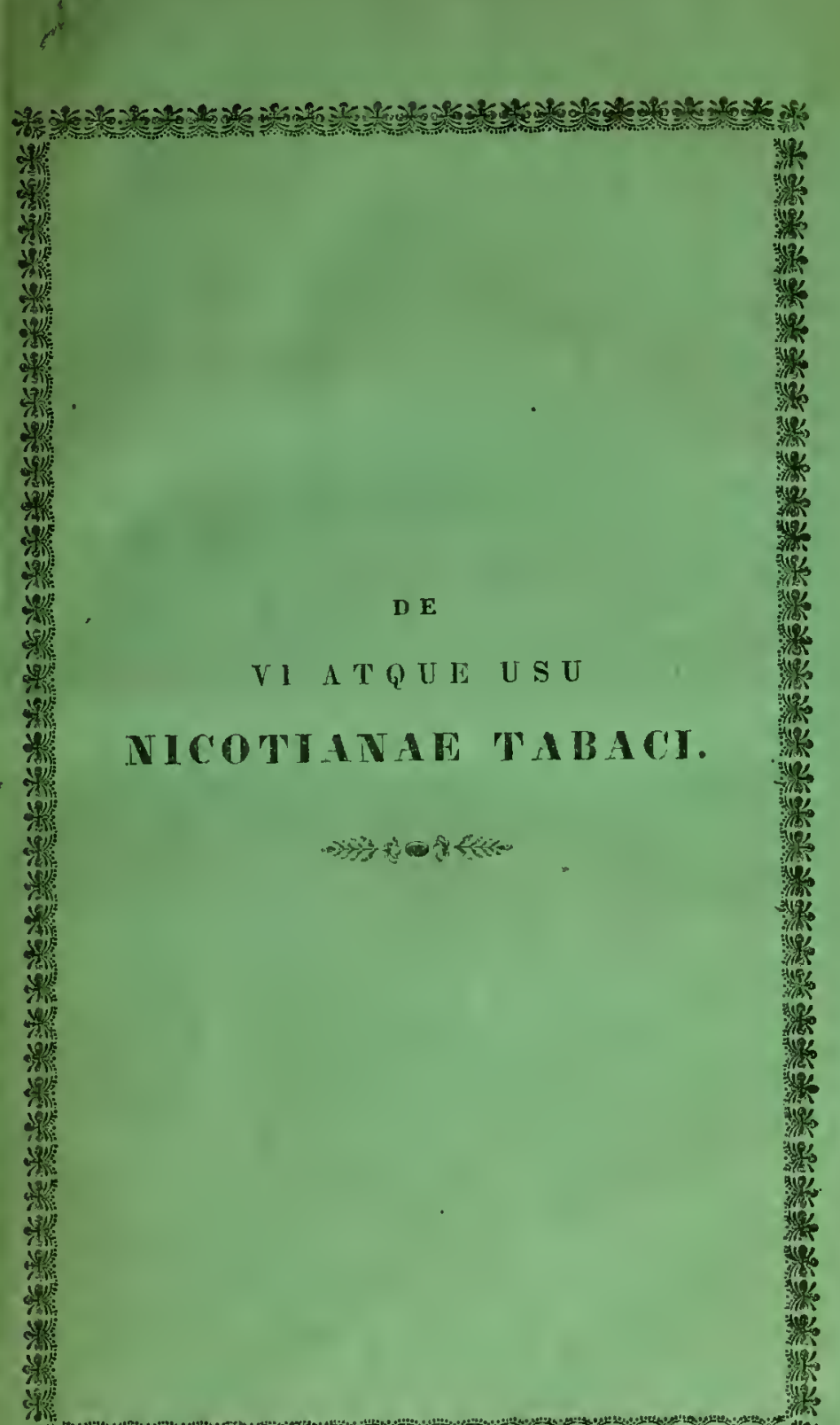

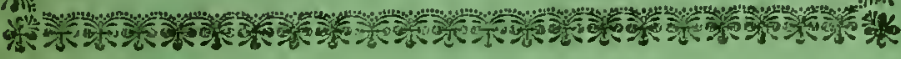




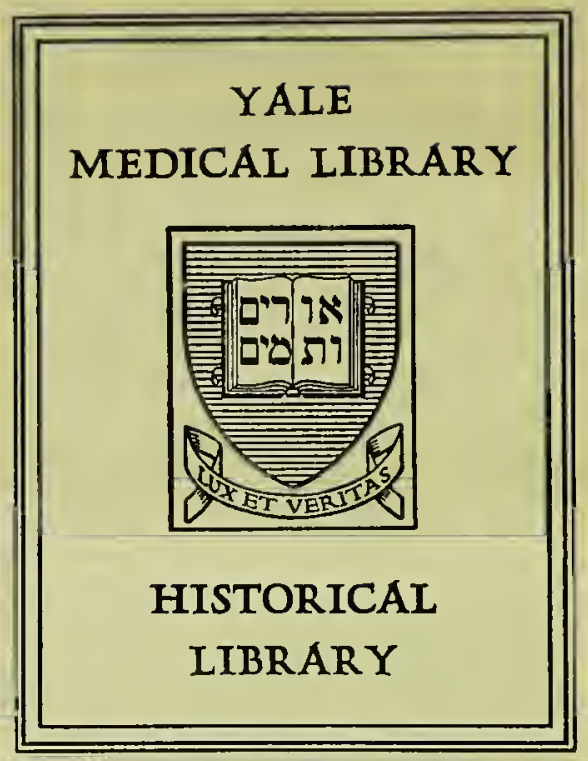

TRANSFERRED FROM

YALE UNIVERSITY LIBRARY 
DE

VI ATQUE USU

NICOTIANAE TABACI.

$\rightarrow 3900<2<-$

DISSERTA'TIO

$\begin{array}{lllllllllllllllll} & \text { I } & A & U & G & U & R & A & L & I & S & M & E & D & I & C & A\end{array}$

Q U A in

A U C T O R I T A T E

G K $\Lambda$ 'I I O S I

MEDICORUM ORDINIS HALENSIS

U $\mathbf{r}$

SUMMOS HONORES

IN MEDICINA ET CHIRURGIA

RITE ADIPISCATUR

DIE VI. SEPTEMBRIS MDCCCXXXVII.

UNA CUM THESIBUS

PUHLICE DEYENDET

AUCTOR

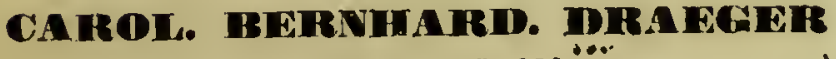
STEINBACHIENSIS

OPPONENTIBUS

JAC. KLINGEBIEL, MED. CAND, HERM. RATH, MED. DD.

\section{I I I}

TYPIS CAROLI GRUNERT. 
symerationen

$R C 567$ 


\section{I R O}

\section{A MPILISSIMO ERUDITISSIMO}

\section{IN'TEGERRIMO}

\section{PETRO KRUKENBERG}

MEDICINAE ATQUE CHIRURGLAE DOCTORI, MEDICTNAE PROFESSORI PUBLICO ORDINAKIO, IASTITUTI CLINICI MEUICI HALENSIS DIRECTORI, REGI BOHTSSLAE

A CONSUIS MEDICIS INTIMIS ETC. ETC.

\section{PRAECEPTORI}

DILECTISSIMO MAXIME VENERANDO 



\section{I R $\mathbf{~} \mathbf{O}$}

\section{HUMANISSIMO DOCTISSIMO}

ADMODUM REVERENDO

\section{DOMINO}

\section{FRANC. HUEHNERMUND}

BAROCHO STEINBACHIENSI, PATRINO DILECTISSIMO

NEC NON

\section{I R O}

ORNATISSIMO HUMANISSIMO

\section{ADAMO FREUNDLIEB \\ SECRETAHIO REGIO TORBIENSI}

\section{A F F IN I}

A M ICISSIMO DILECTISSIMO 


\section{n) $\&:$}

vitic

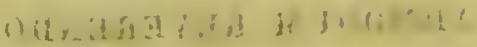

\section{4: iirue:}

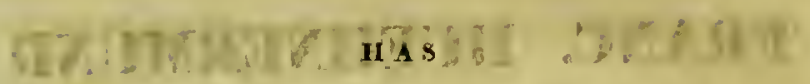

STUDIORUM PRIMITIA S

\section{PIO GRATOQUE ANIMO}

$=131$

D. D. D.

(1) is in

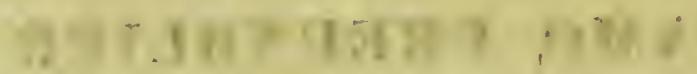

A UCT $\cap \mathrm{R}$ 
Lerbae nicotianae quotannis multa per omnes terras vis luxuria quadam consumitur, sed perperam multi multos inde deducunt morbos, immo nisi immoderate nicotiana utaris damnosa habenda non est. Qua de re nonnulla imperia, quae tabacum importari vetuerunt, omnino recte non fecere, neque, quae in universum tabaci usum prohibuere, neque I a c ob us rex, qui Philippicam quandam contra hanc herbam scripsit, aut Amaradius IV., qui fumum sugere crimen capitale haberi jussit, aut papae, qui eos excommu-; nicaverunt, qui nicotiana uti consueverant; adeo in Russia olim, qui fumum sugebant, nasum perdendi periculum adiebant, atque in pago Bernensi fumum sugere secundum habebatur post stuprum crimen. Neque tamen infitias ire debemus tabacum immoderate usurpatum pro veneno vehementissimo habendum esse atque omnes eius partes quacunque forma et qualibet ratione adhibitas venenorum in modum agere, quamquam ipsa forma atque adhibendi ratione effectus immutatur, fumus enim minus valet, quam infusum, externo lentius usus internus, et ille ninus damnum affert, quam si pe* clysma tabacum in corpus iniicias. 


\section{A P U T I.}

\section{Quomodo organismus noster} tabaco afficiatur.

\section{s. 1.}

Foliorum nicotianàe vapores, si diutius atque e magna tabaci copia orti hominem petunt, facilline vertiginem," maiorem aut minorem stuporem, veternumque efficiunt.

F a u r c r o y etiam narrat, puellam, quae in conclavi dormiebat, ubi multam nicotianam raserant, exortis inde vaporibus, interemtam esse.

$$
\text { \$. } 2 .
$$

In is, qui sugendo fumum nondum assuevere, aut qui iusto plus et diutius sugunt tabacum, facile nausea, vertigo, inclinatio ad vomitum et diarrhoeam, deinde vomitus ipse et diarrhoea, aucta urinae secretio, coëundi cupido, denique lassitudo quaedam et syncope efficitur, quam profundus sequitur somnus.

Dr. Marsh a $\mathrm{Hall}$ adolescentem narrat, qui primum duas pipas suxisset, statim nausea, vomitu, syncope, deinde anhelitu stertente, spasmis universalibus atque pupillae immobilitate affectum esse; postridie etiam inclinatio ad syncopen nondum desierat et cum vesperasceret stupor et stertens respiratio redierunt; 


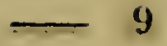

attamen ab boc inde tempore sensim sensimque revaluit. *)

Gmelinus mortem adeo immoderate sugendo fumum effectam esse memoriae prodidit duorum hominum, quoram unus XVII alter XVIII continenter pipas suxerat. ${ }^{* *}$ )

Quamquain qui intemperanter fumum sugunt, perraro tantum mortis adeunt periculum, tamen saepe inde oritur plethora abdominalis et qui hac consuetudine obligati sunt, si vespertino tempore multum suxerunt, paulo ante, quam somnum capiunt, aliquoties pertimescere solent, atque cordis motus is desinere videtur, seseque putant suffocari, quam rem primum cordis palpitatio, deinde tandem somnus sequitur. Vehementius etiam ita ii afficiuntur, qui fumum sugentes theae viridis infusum bibunt, nam haec eandem fere, quam tabacum possidet vim; utraque enim herba cordis nervarum facultatem deprimit.

\section{S. 3.}

Qui pulvere tabaci sternutatorio intemperanter utuntur, ii iisdem fere quam qui sugunt fumum dam. nis obnoxii esse possunt. Namque huius pulveris usu nervi olfactorii facile redduntur insensibiles, nares inflammantur, ulcera in iis peiora excitantur, et qui hoc pulvere uti non solent, iis vehementior inde effecta sternutatio damnum etiam afferre potest. Lanzoni enim narrat, hominem quendam, pulvere sternutato-

*) Edinb. Hed. and surg. journ. Xll. Il.

**) Gmelin Toxicologie, 550 . 
rio intemperantius naribus immisso, in veternum incidisse et post duodecim dies lethargosum esse mortuum.*) Adeo Ch e yn e persuasum habet apoplexiam in iis habendam esse morbis, qui e nicotianae usu proficiscantur, sed haec sententia eorum, qui et fumum sugunt et pulvere sternutatorio utuntur, refutari videtur raletudine. ${ }^{* *}$ )

\section{§. 4.}

In applicatione foliorum nicotianae ad cutem externam summa opus est prudentia; maiorique etiam, ubi laesa et corrosa ; nam tum facillime nauseam, vertiginem, vomitum atque universalis defatigationis sensum ciet, et cum diutius in tales corporis partes nicotiana applicatur, universalis membrorum tremor levesque efficiuntur convulsiones; pulsus fit frequens et debilis, brevique difficilique respiratione postremo consequitur mors. Murra y narrat, tres infantes postquam in vomitum, vertiginem, et multum sudorem incideriut, praeterlapsis XXIV lıoris, mortuos esse, quia mater ad tineam capitis ex qua liberi liberarint linimento tabaci usa esset.

Watterhal similem memoriae rem prodidit. Puer enim tribus horis obiit, postquam in tineam, qua laborabat, succum tabaci infuderant.

Experimenta in animalibus instituta simillimos ostendunt effectus.

I. Columbae enim pastquam in femoris interiori latere ita feci cisionem, ut neque musculi ullius fibra laederetur neque animal sanguinem pederet, ad telam cel-

*) Ephem. cur. nat. Dec. II. Ann. X. p. 222.

**) On apoplexy and lethargy, p.550. 
Iulosam unum fere scrupulum pulveris foliorum nicotianae pharinacopoeae borussicae, paululum aqua madefactorum, applicavi; : quo facto exspectavi, quid inde fieret. Statim luculenta apparuerunt symptomata, reneno organismum affectum esse. Columiba enim anxiam accepit speciem, respirabat alte, post dimidiam minutam paralysi ; utroque pede infracto, corruebat, perquam anxie atque alas valde agitando studebat avolare, aliquoties late aperiebat rostrum, tamquam ad romitum, nec tamen vomuit, neque urina aut faeces eiectae sunt. Simul vero alia totius corporis convulsio aliam sequebatur; caput erat retractum, oculi late aperti, pupilla magna et paene immobilis, rostrum continenter. pauxillum aperiebat, lente et anxie respirabat, pulsus cordis erat frequentissimus. Postremo inquietudo desinebat, alas non amplius columba agitabat, atque respiratione facta lentissima et profundissina, animal praeterlapsis post applicationem tabaci in vulnus quinque 'minutis lévi convulsione obiit. Seclione facta nihil ullius momenti animadversum est.

II. Simile porro experimentum feci in gallo gallinaceo. Huius in femoris interiori latere item facta cisio$n e$, in vulnus scrupulum unum foliorum nicotianae pulverisatoium intuli. Nunc quoque manifesta animadversa sunt symptomata gallum veneno esse imbutum. Statim enim animal lassum exstitit et defatigatum, demisso capite lente altéque respiravit, paralysis secuta est extremitatum inferiorum, serius tamen, quam in priori experimento, pes sinister non laesus paralysi prior infirmatus est. (Omnino cum experimenta in avibus instituerem, priorem sinistri pedis paralysin animadverti, ) 
Animal iacebat perpetuo in latere, vomituritio et faeces separandi studium non apparnerunt. Pulsus cordis frequentissimus, caput. valde ad tergum fuit retractum, pupilla vehementer angusta, iris celeriter mota est. Animal magis magisque defatigari visum neqque mortem non continuo secuturam exspectavi, nihilo. secius post nnam fere et dimídiam horam gallus recreatus cibum capiendi cupidinem prae se tulit, et ex aperto' vulnere nicotiana remota, omnino vires recuperavit.

III. Caniculi in femoris vulnus eadem ac antea ratione drachma una pulveris foliorum nicotianae illata, superiora symptomata non animadversa sunt, nisi quod animal anxium et incitatum visum est, postero tamen mane mortuum erat.

S. 5 .

Interne adhibita pro dosis et formae diversitate varios nicotiana ciet effectus; vehementissime, ut alia quoque multa huius familiae remedia, in intestinum rectum applicitum valet.

Per minores sumpta doses prinum auget secretionem in membrana canalis intestinorum mucosa, efficaciam vasorum resorbentium, ideóque secretionem urinae. Proximus praesertim, cum per infusum nicatiana adhibetur, effectus semper pungens quidam et praeteriens caloris in gula sensus, quem saepe item caloris in ventriculo sensus sequitur, tamquam vini adusti haustum sumsisses. Proximo autem universali minorum dosium effectu urinae augetur secretio, qui effectus modo cum vertigine coniunctus est, modo non; saepe fluxus etiam alvi cietur in universum vertigine, taedio aut nausea 
comite; dolores, qui adsunt, deliniantur; in nonnullis tabaco dormiendi cupiditas efficitur, somnusque augetur; in aliis item cupido dormiendi, cui adiunctus est caloris et inquietudinis sensus; nonnunquam dolores quoque colici, diarrhoea, vomitus, vehemens et universalis sudor, immo capitis dolores aut membrorum tremor animadvcrtuntur. 'Ubi per maiores doses tabacum usurpatur, vomitus vehemens diarrhoeaque cientur. Paulo post haustum remedium magnae sensus inquietudinis, anxietatis atque angustiae in pectore atque regione hypochondriaca evadit; respiratio profunda exsistit et abrupta, deinde magna vomituritio, vomitusque et diarrhoea consequitur, quo facto ingens lassitudo, nervorum relaxatio, debilitas et proprius membrorum, qui numquam deest, tremor nascuntur. Anxietatis cum inclinatione ad deliquium animi coniunctus sensus, quen levium quoque convulsionum saepe comitantur impetus, augetur aliquamdiu; simul pulsus fit parvus, debilis, partes exteriores, inferiores imprimis extremitates, organicum perdunt calorem, iisdem praccipue in membris convulsiones vehementiores oriuntur, quas mox deinde paralysis torporque harum partium excipit. Qui si apparent effectus, pupilla angustior fit, id quod externum etiam tabaci infusum ad oculum applicatum elicit, ut ego saepius in experimentis, quae institui, observavi. Neque memoratu indignum est, paralysi inferiores tantum fere semper extremitates affici; nervi sensorii illaesi sunt ct motorii tantum infirmati.

Neque tamen simul sensus organa valde afficiuntur; animalia enim, in quibus institui cxperimenta, alacrem semper neque tristem plerumque prae se tulerunt vul- 
tum, rebusque externis commota eas evitare studuerunt, quamquam veneficii symptomata clarissima apparuere. Qui ubi paralysis status, quem continenter vomituritio saepeque etiam ipse vomitus comitantur, longius breviusve temporis spatium duravit, profundus sequitur continuusque somnus, qui ut desiit veneficii quoque symptomata paralysisque evanuerunt, neque nisi lassitudo quaedam defatigatioque totius corporis, quae et ipsae mox desinunt, animadvertuntur.

Quodsi doses etiam augentur ad $3 \mathbf{v}-\mathbf{v r}$, symptomata superiora multo citius vehementiusque ex sistunt atque post longius breviusve tempus universali corporis tremore, quem paralysis extremitatum antecedere solet, et profunda operosaque respiratione sequitur mors. S a n te uil, vir celeberrimus, vomitu vehementibusque doloribus obiisse dicitur, quod vinum biberat cui tabacum immixtum fuerat. *)

Fautrel hominem mori se vidisse narrat, qui quantum cochlear capit, tantum succi hauserat nigri, qui oritur, cum fumum sugunt. ${ }^{* *}$ )

Quae antecedunt his demonstrari videntur experimentis.

I. Orfil a enim cum rentriculo canis satis robusti 3vß tabaci intulisset, ligatura oesophago circumdata, paucis minutis post vomere animal contendit, incessus lentior factus est, vertigo etiam et continuus inferiorum extremitatum tremor ortus, sensus organa vacua esse

*) Orfil a toxicologie gien. II. 280.

**) Journal de chimie médicale de pharmacie, de tosicologie et revue des nouvelles scientifiques nationales et étrangères. Nro. VII. tom. IV. II. Série Juillet. p. 317. 
visa, respiratio autem paulo concitatior; post paucas horas animal in latus stratum, quoniam non amplius stare valebat, caput continenter tremuit, nuchae musculi levi convulsione commoti, membra languida ; physiognomia stuporis speciem ostendit, respiratio profunda, laboriosa, concitata, cordis pulsus frequens, paulo fortior fuit et post IX horas, quam tabacum iniectum erat, animal mortuum est.

II. Item $\bar{\zeta}_{1}$ siccorum nicotianae foliorum una cum

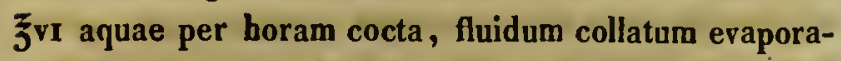
tione ad quatuor et dimidiam unciam redactum est. Quo facto cum fluidum ventriculo canis bene nutriti, mediae magnitudinis, iniectum esset, ligatura oesophago circumdata, post tres minutas animal vomiturum esse visum, idque per primam horam saepius repetuit, deinde initio extremitates posteriores vehementer ceperunt tremere, id quod paulo post in omnibus membris animadversum est, quae deinde vicissim convulsionibus vehementioribus pressa sunt, et tribus horis post illatum fluidum animal obiit.

Facta utriusque animalis sectione, ventriculum levius narrat $\mathrm{Or} \mathrm{f}$ il a fuisse inflammatum, pulmones lividis conspersos maculis telaque magis compacta, quam firma valetudine esse solent. *)

III. Equidem cum pullae columbae quatuor grana extracti nicotianae ventriculo intulissem, atque ne vomitu extractum eiiceretur, ita cavissem, ut nostrum obligarem, quamquam columba per plures minutas insensibilis manere videbatur, tamen eam non bene ha-

\#) Orfila toxicologie, II. 
bere animadverti, quieta fuit saepiusque oculos clausit. Paulo postea vomituritio orta, brevibus intervallis repetita; deinde postquam praecipitavit columba, primum sinistri, tum dextri quoque pedis paralysis consecuta est, neque vomituritio desiit. Simul omnia membra tremuere, animal valde fuit inquietum vicissimque avolare studuit. Organa sensus illaesa fuere. Postremo post horae quadrantem vomituritio desiit, animal defatigatum et lassum fuit, cordis pulsus frequentissimus, et post quinque horas columba levibus convulsionibus periit. Sectione instituta, gravius nihil repertum; extractum nicotianae adhuc maximam partem in gutture adfuit praecipue pisis adhaerens, quae columba pasta erat, in ventriculo et intestinis extracti vestigium non observatum.

$$
\text { \$. } 6 .
$$

Validissima perniciosissimaque, ut iam supra memoravimus, nicotiana est; ubi per clysmata usurpatur; quam quidem in formam redacta et plerumque adhibetur et si providus reique medicus peritus ea utitur, saluberrimum est remedium. Sive fumum, sive decoctum, sive infusum clysterizando adhibes, omnino veneficium paralysisque aeque apparet; infusi autem effectus vehementissimi sunt, statimque post applicitum clysma animadvertuntur; neque tamen negligendum est clysmatum tabaci effectus, quamvis candem formam dosimque conserves, pro aetate, corporis constitutione, genere etc. esse diversos, consuetudinemque magni esse momenti; qui iam dudum fumum sugere consueverant et robusti homines, non tantopere afficiuntur clysmatibus, quam quorum corpus fluxum et debile, 
quique tabaco non adsuevere. Alius in alio tempore effectus animadvertitur, et quamquam in aliis parvae ipsae doses lethales sunt, in aliis multo maiorum levis tantum effectus. Quae in universum clysinata, si minores adhibeas doses, (i. e. si unius sive duarum drachmarum, quibus aq. $\tilde{5} x$ infusae sunt, dimidiam partem corpori iniiceas) phaenomena sequuntur, haec fere sunt:

$\Lambda$ egroti mox de anxietatis quodam per totum corpns divulgatae sensu queri incipinnt; dolores, quibus cruciantur, statim interdum amittuntur; simul defatigatione quadam obruuntur, verliginem, romituritionem vomitumque etiam ipsum, leves imprimis in abdominis musculis convulsiones, inclinationemque trahunt ad deliquium animi; respiratio difficilis est et profunda, pulsus rariores et sudores frigidi, quo facto aegroti profundum tranquillumque capiunt somnum, quo praeterlapso haec quoque symptomata evanuisse solent. Sin autem dosin auctiorem (dimidiam sive unam circiter unciam) aut minores doses supra dictas, iusto pluries brevioribusque intervallis adhibeas, aut ubi acgrotus talis est, ut facile doleat: tum quae supra memorata sunt phaenomena, multo apparent vehementiora. E vestigio interdum magnae convulsiones oriuntur, quas aut ad syncopen inclinatio aut ipsae comitantur eneque continuae syncopae; sinul rultus pallescit et collabitur, pulsus fit parvus, debilis, frequentissimus; cor palpitat violenter, respiratio difficillima est et profunda, et postremo spiritu profundo, breviori et abrupto et universali membrorum tremore celeriter accedit mors.

Veneficiorum hac re tabaco factorum exempla mul- 
tis in libris et antiquis et recentibus reperiuntur et quotidie etiam facile eiusmodi res observes.

Astley Cooper hominem narrat, cuius ad herniam incarceratam infusi nicotianae clysma, paratum ex $\mathbf{I I}$ adhibitum erat, cum post dimidiam horam hernia nondum recessisset, ad instituendam operationem in mensam esse prostratum; sed quia pulsus debilis vultusque collapsus fuisset ct corpus multo sudore frigido coopertum animadvertissent, aegrotum in lectum reportatum esse, qua in re obiisset. ${ }^{*}$ )

Item puellae, quae et ipsa hernia laborabat incarcerata, infusum unius drachmae tabaci per clysterem cum esset immissum, vehementes in abdomine dolores et vomitus sunt consecuti, quo materiem eiecit, quae tabacum valde oluit, atque triginta quinque minutis post clysmatis applicationem mortua est, quae quidem mors tabaco verisimile effecta est. ${ }^{* *}$ )

Anonymus quidam in Actis Helveticis scriptor memoriae prodidit feminam, quae clysma unius unciae accepisset, doloribus in abdomine, anxietate inclinationibusque ad syncopen affectam, paucis horis praeterlapsis morte occubuisse. ${ }^{* * *}$ )

Ad Journ. chimie médicale femina clysmo, qui ex $\bar{\zeta}^{1}$ tabaci ad iufusum paratum erat, quinque minutis interemta est. ${ }^{* * * *}$ )

*) Astley Cooper Anatomie .und chirurg. Pehandlung der Leistenbrüche und der angeborenen Brüche. Aus d. Engl. Breslau " 1809.

**) Ebendaselbst.

***) Acta Helvetica. 1762. V. 330.

***) Journal chimie médicale, Tom. 3. pages 23 el 592. 
E narratione anonymi scriptoris in Med. and surg. journal homo, quem curabat, clysmate obiit post duas horas, quod ex $\mathbf{3}$ II foliorum tabaci quibus $\bar{\zeta}$ nur aquae infuserat, erat paratuin.*)

Similia invenies in: Journal. Edinb. med. und surg. tom. 36. pag. 227. Huf el and s Journ. d. prakt. Heilkunde, L. XXI. IV. 100.; ihid. vol. 24. pag. 108. (1810.); |ibidem vol. 45. fasc.IV.pag. 109. (1807.)

In Journal de chimie médicale de pharmacie, de toxicologie, et revue des nouvelles scientifiques nationales et étrangères. No. VII. tom. IV. Serie II. Juillet. Paris 1838. recens exponitur veneficium pluraque vetera adduntur.

Veneficium clysmo tabaci effectum, quod dimidio abhinc anno hic ibidem accidit iam prolixius exponatur.

J. St., puer robustus, XXI menses natus $\mathrm{XV}$ iam menses hernia inguinali laboraverat, quae ultimo tem. pore raro tantum prodierat semperque sponte et facile redierat. Praeterlapsi anni XXI die mensis Novembris hernia iterum magisque, quam antea prodit, neque sponte recessit, et sequenti die omnia incarcerationis apparuere symptomata. Parentes autem, qui sperarant, fore ut morbus cura medica non adhibita sedaretur, XXIII. demum die Novenıbris, postquam incarceratio tres fere dies duravit, auxilium ab hoc clinico medico petierunt. Puer iam tres dies exoneratione alvi non gavisuš, paülum ederat, solito vero plus biberat saepiusque in calorem inciderat, vomueratque neque tamen faeces einișerat. Abdomen expansius repertum

*) Edinl. med, and surg. journal. IX. 159. 
est, calorque eius moderatus, neque pressu externo animadversum est dolorem effici, et urinae secretio'inturbata fuit. Herniae ipsius calor moderatus' itemque ruber fuit, atque in universum incarceratio non acutior. Simul universa valetudo bene habuit, neque febris fuit gravior.

Frustra repositionis periculo facto, sè hérniae admoti sunt hirudines pluresque saponis clysnios brevibus intervallis adhibere iussum est, quos, si exoneratio alvi non sequeretur, tum clysmata infusi foliorum nicotianae applicari; idque ita, ut uni drachmae foliorum tabaci octo unciae aquae infunderentur atque hoc infusum tribus dosibus iniiceretur. Mater, quae de clysmatis tabaci audierat, cum obstetrice, quae saponis clysmos applicuerat, quum hos exoneratio non statim sequeretur, se audiisse communicavit tabaci clysteres esse adhibendos, si saponis clysmata bonos non haberent eventus, medicum vero ipsum rei interfuturum esse. Sed obstetrix, ubi matri se saepius iam tabaci clysmata applicuisse confirmavit, neque medici hominis ad hanc rem opera esse opus, postquam tabacum parari iussit: mater, quae obstetriciis verbis maxinıo cum suo dolore fidem haberet tabaci, haud valde pretiosi, uncias fere unam et dimidiam afferandas curavit, unde cum funesta obste: trix adhibita aqua decoctum fecisset, , clysma huius fluidi iniectum est.

Clysmate vix applicato puer brachiis pedibusque vehementer contremere, profunde aliquoties respirare, contorquere oculos, vultus perfundi pallore atque duabus post clysmatis applicationem minutis his symptomatibus morte occumbere. Sectio non est permissa. 
Lixperimenta instituta in animalibus.

I. Brodie in complurium canium et felis intestinum rectum $\overline{\mathfrak{j}}^{\mathrm{l}}-$ Iv fortioris tabaci infusi inspersit. . Quo facto animalia paralysi affecta, omnia nondum praeterlapsis decem minutis mortua sunt. Pulsus cordis una ante mortem minuta, non iam animadvertendi fuisse traduntur; unum modo animal vomuit. Sectione statim post mortem instituta, cor repertum valde extentum neque amplius se contraxit; semel tantum cordis auricula instrumentis irritata contrahi coepere, item circulatio aërem iuspirando in pulmones dimidiam horam sustentari potuit.

II. Equidem infusum e 311 foliorum nicotianae, quibus quatuor uncias aquae fervidae infuseram, paravi; post quartam deinde horae partem in columbae intestinum rectum scrupulos duos inspersi. Animal, ubi consedit, scrupulus fere dimidius infusi una cum faecibus eiectus est. Columba initio inclinationem exonerationis alvi ostendit, tristissima, anxia et inquieta evasit, alas agitavit, oculos ad dimidiam partem clausit et post tres fere ninutas vomere coepit. Sex minutis post iniectum clysma derepente sinistro pede porrecto collapsa, et mox inde utriusque pedis paralysi allecta est. Iacuit in alvo, ambo pedes porrecti et intenti, minorem quam ceterae corporis partes organici caloris gradum habuere. Vo= mituritio simulque ad exunerandum alvum inclinatio duraverunt, neque tamen faeces emissae.sunt. Oculi aperti, pupilla paulo angusior fuit, iam intervallis quinque fere minutarum unicersi corporis tremor ortus est, atque pennae inprimis in capite erectne sunt. Postquan animal hanc paralysin borae dodrantem tolera- 


\section{$-22$}

vit et alae etiam infirmatae sunt, aliquoties faeces eiecit fluidas, qua de re tabaci etiam infusum eiectum ést, et mox inde vires reconciliavit. Paralysis transiit et columba breve tantum 'etiam tempus anxiam habuit tristemque faciem; sed certe obiisset, nisi corpus nocens esset remotum.

"III. Aliud cum facerem periculum $\xi^{\prime}$ eiusdem, quam. antea memoravi, formae usque ad $\mathbf{J}$ IIr decoxi et huius fluidi ' $\boldsymbol{F}_{\mathrm{I}}$ in intéstinum rectum columbae inspersi, sed' ano ligaturam applicui. Paulo postea, ut in experimento superiori vomitus, exonerationis alvi studium; extremitatun paralysis secuta sunt, simul respiratio difficilis et profunda, cordis pulsus frequens, pupilla paulo angustior exstitit, deinde universalis secutus membrorum tremor atque post duodecim horas, quamquam ani ligatura remota erat et columba aliquoties emiserat faeces, universalibus convulsionibus mortua est. Sectio, quam deinde institui, nilil praebuit cognitu dignum.

IV. Paravi ex $\bar{\zeta} ß$ foliorum tabaci et $\bar{\zeta}$ r aquae fervidae infusum, cuius $\bar{j}^{\mathrm{nt}}$ in pullae felis intestinum rectum intuli et nunc quoque ligatura usus sum. Post duas fere minutas in omnibus membris vehementer coepit tremere, pedibus stare non iam valuit, respiratio brevis et profunda, cordis pulsus frequentissimus et debilis factus est, et post sex minutas convulsionibus levibus animal mortuum est. Sectione facta nihil memoratu dignum inventum est. 


\section{$-23-$
CA P U T II.}

\section{De providentia, qua in adhiben-}

\section{di) tabaco opus est.}

Ex iis, quae hucusque exposita sunt, satis patet, nicotiana organismum humanum vehementer affici eamque, quacunque in forma et minoribus etiam dosibus vehementis veneni vires praebere, ideoque liuius her-' bae in usu maxima opus esse cautione, si malos erentus impedire velis.

Internus remedii usus ceterum nondum valde divulgatus rarissime malos effectus ciet, quamquam semper dosibus pro rerum conditione minoribus initium facias -portet, easque sensim sensimque tantum adaugeas; saepe enim homines invenimns, qui nicotianam aut difficile aut omnino non tolerare possunt, et in quibus e restigio reneficii symptomata excitat. Infantes et caduci homines minus tabacum sustinent, quam adulti et robusti; atque Fowler se dicit raro hoc remedio in infantibus, qui quintum annum nondum explevissent esse usum, quia in his effectus non manifesto perspiceretur, fortiusque iufantes nicotiana afficerentur. *) Semper in usurpanda interne nicotiana observandum est, ut remedium simulac aegroti de vertigine, anxietate, inquietudine, vomituritione et ad diarrhocam inclinatione doloribusque etiam colicis queri incipiunt, statim re-

*) F. Towler, ron den Wirkungen des 'Tabaks, in den ausorlesenen Abhandlungen. Bd. XI. St. 3. p. 399. 
iiceas, neve priusquam baec symptomata trasierint, ad nicotianam redeas. Praeterea doses ad las res instituendas esse, vix dicere opus est. Conf. $\$ 5$.

Ubi tabacum externe adhilies, cavendum est, ne corrosis laesisque cutis locis, ulceribus etc. applices. Hoc ubi fieri debet, caveas, ne remedium iusto longius insit ulceribus cet., quia hac ratione facillime vehementissimi et perquam periculosi eventus, ipsa etian mors cieri possunt, nam exceptis clysmis, si remedium cuti externae applicas, efiectus vehementissimus est. Conf. \$. 4 .

Tabacum, quoniam per clysmata applicitum validissimum est, utque hac ratione pleraque fiunt veneficia, medici est, ubi his clysmis utitur praecipuam provideutiam adhibere.

\section{Iam necesse est:}

J. medicum tabaci speciem, unde clysma parandum est,

II. dosis moduin,

III. formam in qua clysma usurpari vult, clare indicare;

IV. ipsum clysmatis, si fieri potest, applicationi interesse, ut eins effectus observare atque ex iis, qua porro opus est, dirigere possit operam.

Quod prinum locmu attinet, optime ea tabaci species, quae in pharmacopoea nostra indicata est, usur-. pari videtur; solita enim nicotiana sugi et cui insculum iam additum est, eius effectus vehementer varii sunt; modo nihil efficit, modo vehenentia, quae nervorum morbum aut irritationem canalis intestiualis inflanmatorian significant, syınptomata efficit. 
Quantum ad dosin, maxima opus est providentia, et cavendum, ne iis, , quae in multis leguntur compendiis, ubi ingentes a nobilissimis medicis tabaci copiae imperantur, exempli gratia a celeberrimo Richter, ${ }^{*}$ ). qui unciam unam cui unciae duodecim aquae infusae: sint, adhiberi vult, incitemur, ut similia faciamus, nisi post funestissimos eventus perperam te egisse intellige-, re velis. In universum statuendum est, neque tamen. unquam haec dosis superanda est, infusi foliorum nicotianae e drachmis duobus et duodecim unciis aquae, dimidiam partem per clysma adhibitam, ubi adultum ceterumque sanum liominem, cuius complexio robusta est, curas, dosin esse idoneam ; sin autem infantibus, aut debilibus mederis hoininibus dosin minorem esse necesse est; hic potest infusi ex una drachma foliorum nicotianae et unciis decem aquae, dimidia pars per clysma adhiberi. Hac ratione clysmata tabaci in nostro nosocomio medico ex multis annis optimo cum eventu adhibentur, neque celeberrimi medici de hoc usu aliter sentiunt.

Percivall Pott infusum ad clysmá adhibendum e pinta fervidae aquae et drachma una tabaci parandum curavit. ${ }^{* *}$ )

Astley Cooper clysma infusi nicotianae ita parari iussit, ut uni drachmae tabaci duodecim unciao aquae fervidae infunderentur, quo quidem infuso decem minutis postea usus est. At, inquit, cum validi huius remedii effectus pro aegrotorum constitutione sit alius atque tabaci etiam natura forsitan alicuius sit momenti,

*) Anfangsgründe der W'undarzneylunst. Bd. 5.' S. 264.

*) Percivall Pott's chirurgische Beobachtungen, aus dem Engl. übersetz.t. Berlin 1776. S. 104. 
initio oportet dimidia tantum pars iniiceatur dimidiamque post boram altera, $:$ si alteram dosin eventus, qui satisfaciat;' non consecutus est. Ii, qui ex aliis audierunt duaram drachmarum infusum, recte una quidem iniectione aegroto inferri, quam supra dixi, cautionem superfluam habeant; sed ego pro meis observationibus confidenter possum affirmare, e praecipite fortioris infusi usu effectus posse proficisci, quorum medicum vehementer poeniteat. ${ }^{*}$ )

In universum haec providentia iusto videtur maior, si effectus respicias, qui tale infusum et in animalibus et in hominibus quoque robustis ceterumque sanis sequuntur. Sine dubio mali eventus non sunt pertimescendi, dummodo universam aegroti conditionem bene examines atque cures, si infusi formam supra praescriptam adhibes.

De clysmatis forma in universum differentiam fortasse non statuas; sed si fumum adbibes, effectus lenliores sunt, usus proprium requirit apparatum, praeterea dosis numquam accurate definire potest ab aegroto accepta, quocirca melius est infusum adhibere, cuius praeterea fortior est certiorque effectus atque usus nunc quidem universus. Lassitudinis, sudoris frigidi, ad syncopen inclinationis symptomata, adhibito infuso multo facilius apparent, quam ubi fumo uteris, neve unquam haec symptomata ominosa ducas, contra salutaria sunt haec phaenomena, dumne necessariam cautelam negli-

*) Astley Cooper, Anatomie und chirurg. Behandlung der Leistenbrüche und der angeborenen Brüche, aus d. Engl. Breslau 1809. 
gas, neve ad aliam transeas remedii dosin, priusquarn illa symptomata praeterlapsa sint.

Quae si omuia in adhibendo tabaco semper observas, nisi fausti e remedio eventus non proficiscentur.

\section{CAP UT III.}

\section{De tabaci usu.}

Exceptis clysmatibus, quamquam nicotiana non leves habet effectus, atque. a claris tentata est medicis, neque sine fructu, usus tamen huius herbae nondum est valde divulgatus.

Fowler, ${ }^{*}$ ) qui plurima accuratissimaque tabaco instituit experimenta, hoc remediun, quamquam universalius commendat ad liydropem, nisi torpidus atonicusque huius morbi est character aptum non est, atque vehementer caveas, ne hoc remedium in is hominibus adhibeas, qui propter organicum cordis vitium hydrope laborant, nam cum in hoc morbo cordis nervorun vires iam sint oppressae, hoc remedium infaustissimi eventus consequi possunt. Ceterum Fowler in XXI hydropicis morbum tabaco expellere studuit, et ter tantum remedium irritum fuit.

Fowler, cum suum infusum adhiberet, a minoribus sensim ad maiores doses transiebat, interea etiam extractum gr. I - II p. d. adbiberi potest. In dysuria

*) Fowler, medical reports of the effects of tabaca. Lond. 1785. 
quoque pluries suo infuso optimo cum fructu usus est. "Ut universe, inquit, loquar, infusum tabaci in dysuria validum apparet sedansque dolorem et urinam pellens remedium, , quod vias uriniferas amplificat et in dysuria, grisea aut lapidea materie effecta, eius abitum expedit."

In diversis colicae casibus unam unciam sui infusi per clysma adhibendam commendat, si adultum solita corporis constitutione curaș. Ad eundem auctorem clysmis propter dolorem deliniens eorum taediumque excitans ac relaxans lingenium vasorum systema et propter vires irritantes intestinum quoque rectum bene afficitur, unde celerem levationem eiectionemque faecium efficiunt. Praeterea tabacu'n commendat in exanthematibus scorbuticis, affectionibus catarrhalibus, in asthmate, ubi Roberthus quoque $\mathbf{P}$ age hoc remedium cum fructu adhibitum commendat. ${ }^{*}$ )

Pertinax tympanitis intestinalis, quae per integros duos annos omnibus medicaminibus restiterat, tabaci clysmatis; quae paulo supra dictis in colica usurpatis fortiores erant, omnium cum admiratione levata est.

John Vetch ${ }^{* *}$ ) tabacum commendat in diversis inflammationibus rheumaticis et arthriticis, quibus membranae synoviales affectae sunt, atque confirmat hoc remedio non solum deliniri dolorem inflammationemque. levari, sed ita etiam membra aegrota vehementer adiuvari, ut tonus iiṣ viresque reddantur.

In nervorum morbis digna videtur nicotiana quae.

*) Roberth Page, ïber den Nutzen des Tabalss in einigen Fällen, wo er gewölınlich nicht angewendet wird, in den auserlesenen Alhandlungen. Bd. 7. St. 1. S. 223.

**) Medico-chirurgic.:transactions. Tol. XVI. P. Il. S. 356. 
saepius usurpetur, quam adhuc factum est; neminem enim tabaco nervorum intentionem minui effugere potest.

In neuralgiis capitis, affectionibus hystericis $\mathbf{F}$ ow ler tabacum cum fructu adhibnit. In tussi convulsiva, cum extractum tertia quaque hora (grana duo) usurparentur, optimum apparuit remedium.

Corrrie*) nicotiana ad chronicam vesaniam usus est, passimque etiam tabacum contra epilepsiam usurpatum est. Courrie epilepticos ita a se sanatos esse dicit, ut nicotianae foliorum cataplasma. regioni eorum hypochondriacae applicaret, qua in re clysmata quoque tabaci non erant irrita.

In tetano morbo, qui hucusque omnibus remediis pertinacissime restitit, quo in morbo imprimis Ander : son tabacum commendat, accuratiori dignun videtur animadversione.**) Anders on cum primum huius remedii faceret tentamen ad trismum traumaticum servae aethiopis, forte recentium nicotianae foliorum decoctum adhibuit, quo dimidia quaque hora malas collumque et pectus fomentanda curavit, deinde cataplasmata foliorum nicotianae in maxilla inferiori colloque imponi iussit. Balnea calida, quibus tabaci quantum satis illatum erat, tertia quaque hora repetita atque duo

*) Praktische Bemerkungen über Geisteszerrüttung, .. J Oseph Mason Cox. Aus dem Englischen von Reil. Halle 1811.

**) Ueber den Gebrauch des Tabaks im Tetanus, v. Thomas Anderson; übersetzt ans d. Engl. in den 'auserJesenen Abhandlungen. Bd. 8. St. 3. p. 462. n. Ed. 10. St. 1. p. 141 . 


\section{$-30$}

clysmata eiusdem iusculi intra XXIV horas applicita sunt. Praeterea alvus oleo ricini aperta est, neque fumisugium aegrotae antea ereptum quam vellet. Hac medendi ratione soliti tabaci eventus apparuere, quamquam non tanti, quanti in hominibus, qui firma valetudine gaudent. Defatigatio, lassitudo, universali perspiratione indicata, quae antea localis fuerat, primum est effecta. Aegrota hocce modo sanata est. In alia femina aethiope, quae in tetanum vulneratione inciderat, calidam foliorum nicotianae fomentationem vulneri applicuit, laxans e ialapa et hydrargyro múriatico miti adbiberi atque e forti decocto foliorum nicutianae recens decerptorum balnea parari iussit, quibus aegrota aliquoties per diem uteretur. Aethiops in quoque balneo retenta est, dum taedii sensus vehemensque universalis transpiratio excitaretur; haec medendi ratio usque ad sanitatis reditum continuata est.

Praeterea duos memorat casus, ubi vicissim simile ratione balnea e tabaco parata et clysmata decocti foliorum nicotianae fomentationesque pectori etc. applicitas cum fructu usurpavit.

Andersou se animadvertisse contendit, post magis continuum hunc tabaci usum vehemens valdeque pruriens in capite et collo exanthema exsistere, quod quidem faustum habet indicium.

Haec vero res ex Andersonii observatione in balnearum clysmatumque e quocunque tabaci specie paratorum usu notatu dignissina est, collapsum nimirun hominis tetanici difficillime effici, qui quidem effectus facillime celerrimeque in sanis hominibus excitatur. Quocirca ubi ad tetanum tabaco uteris specifica remedii 
quantitas negligenda' est atqृue solus in usu effectus respiciendus. Ex Andersonii sententia in universum haec sequenda est lex, ut IV libr. herbae siccae unam horam in octo gallonibus aquae coquantur atque haec in balneam tepidum infundantur. - Praeter hos morbos tabacum in aliis etiam rnultis morbis partim adhibitum, partim commendatum: est, quibus brevitatis causa omissis ad usum primarium, ad usum nimirum tabaci clysmatum in herniis incarceratis transimus. Ut plerumque atque in universum fere ineptissimum est ad hernias incarceratas laxantia atque drastica etiam fortiora usurpare, sic contra curatione ceterum iusta atque idonea e clysmatis optimi fructus percipi possunt, atque in hac morborum specie tabaci clysteres multo magis, quam adhuc factum est .adhibendi esse videntur. Rarissime remediumt empore opportuno iustaque ratione adhibitum frustrabitur, immo saepe chirurgum cultro operationem perpetraturum voluptate chirurgica privat atque multo lenius id praestat, quod chirurgi culter, cuius manus maxima habilitate excellunt, praestare non potest.") Quamvis ab hoc quoque passim remedio destituamur, hoc Aesculapii mysteriis initiatus parum admirabitur, cum idem in aliis remediis, quorum contra certos morbos efficacia ab omnibus agnita est, quotidie animadvertamur.

Remedii effectus, si adbibetur in formam redactum cap.II. significatam, cognoscitur debilitate, quam ciet; oriuntur sudores frigidi, pulsus debilis fit et frequens, vertigo, vehemens vomitus, muscularum abdominis convulsiones, syncopes etiam impetus existunt; deni-

*) Percivall Pott's chirurgische Beoabachtungen. 
que tantam membrorum efficit defatigationem, ut aegrotus musculum; qui voluntati obnoxius est, non valeat commovere. Tali exorto universali collapsu hernia plerumque aut sponte aut levi pressu in abdomenureiicitur, quamvis antea multo maiorem vim superaverit.

Saepe plures tales clysmi applicentur oportet, priusquam eventus appareat; immo Percivall Pot t ji tabaci a se esse consumptum narrat, priusquam quod voluit efficeretur atque nonnisi applicata uncia altera solita symptomata apparuere; aegroti de motu in abdomine herniaque queri coepere atque tunc brevi fere tempore hernia rediit. Qua de re exoptatus eventus desperandus non est, si non effectus remedii e vestigio apparet. Certe si doses iusta adhibita cautela repetantur, effectum non desiderabis. Plura exempla, ubi difficillimae diversissimaeque incarcerationes tabaco sublatae sunt, exposuit Percivall Pott. 


\section{I T A.}

Ego Carolus Bernhardus Draeger, confegsioni eatholicac addictus, anno MDCCCXIV. die XIII. Maii, patre Ioanue, matre Elisabetha Dorothea e gente Dcgenhardt, quos parentes dilectissimos iam defunctos vehemeuter lugeo, Steinbachii pago Eichsfeldiaco natus sum. In gymuasio Heiligenstadiensi, quod Directore Cl. Rinke floret, primis litteris per septem annos imbntus, litterarum universitatem Halensem adii et in eivinm academicorum numerum anni MDCCCXXXIV. die XI. Norembris t. t. Rectore magnifico Cel. G e rmar, Decano spectatissimo, Cel. Kruke ab érg réceptus, sum.

Per quadrienuium hisce praelectionibus interfui; Cel. Gerlach de logice, de psychologia; Cel. Schweigger de physice, de chemia; Beat. Nitzsch de zoologia, de helminthologia; Cel. Germar de mineralogia; Cel. de Schlechtendal 'de hólanice; Cel. d'Altón de auatomia, arte cadavera rite dissecandi, ac physiologia; Cel. Friedlacuder de materia medica; Cel. Krukenberg de pathologia atque-therapia tam gellerali quam speciali; Cel. Niemeyer de arte obstetricia; Cel. Blasius de chirurgia geuerali atque speciali, de fracturis atque lıxationibus, de ophthalmologia et de arte fascias applicandi.

Scholas clinicas, medicam directore Cel. Krukenberg, per bicunium, chirurgico-ophthalmiatricam directore Cel. Blasi us, per tria semestria, et olstetriciam directore Cel. Nicmeyer per semestre frequentari.

Quilus omuibus de me maxime meritis riris habeo atque semper maximas gratias habebo. 


\section{T $\quad$ H $\quad E \quad S \quad E \quad S$.}

\section{I.}

Omnes nicotianae tabaci partes sunt venenosae.

\section{II.}

Hernia incarcerata remedia purgantia vetat.

\section{III.}

Blennorrhoea nil nisi exitus inflammalionis.

\section{IV.}

Scrophulosi nulla dyscrasiá saepius invenitur.

$$
\text { V. }
$$

Tunica oculi coniunctiva certe ad membranas referenda pituitosas. 


\section{Accession no.}

9339

Author

Draeger, C.B.

De vi atque usu nicotianae tabaci. Call no.

-19th cent.

$3=567$ 


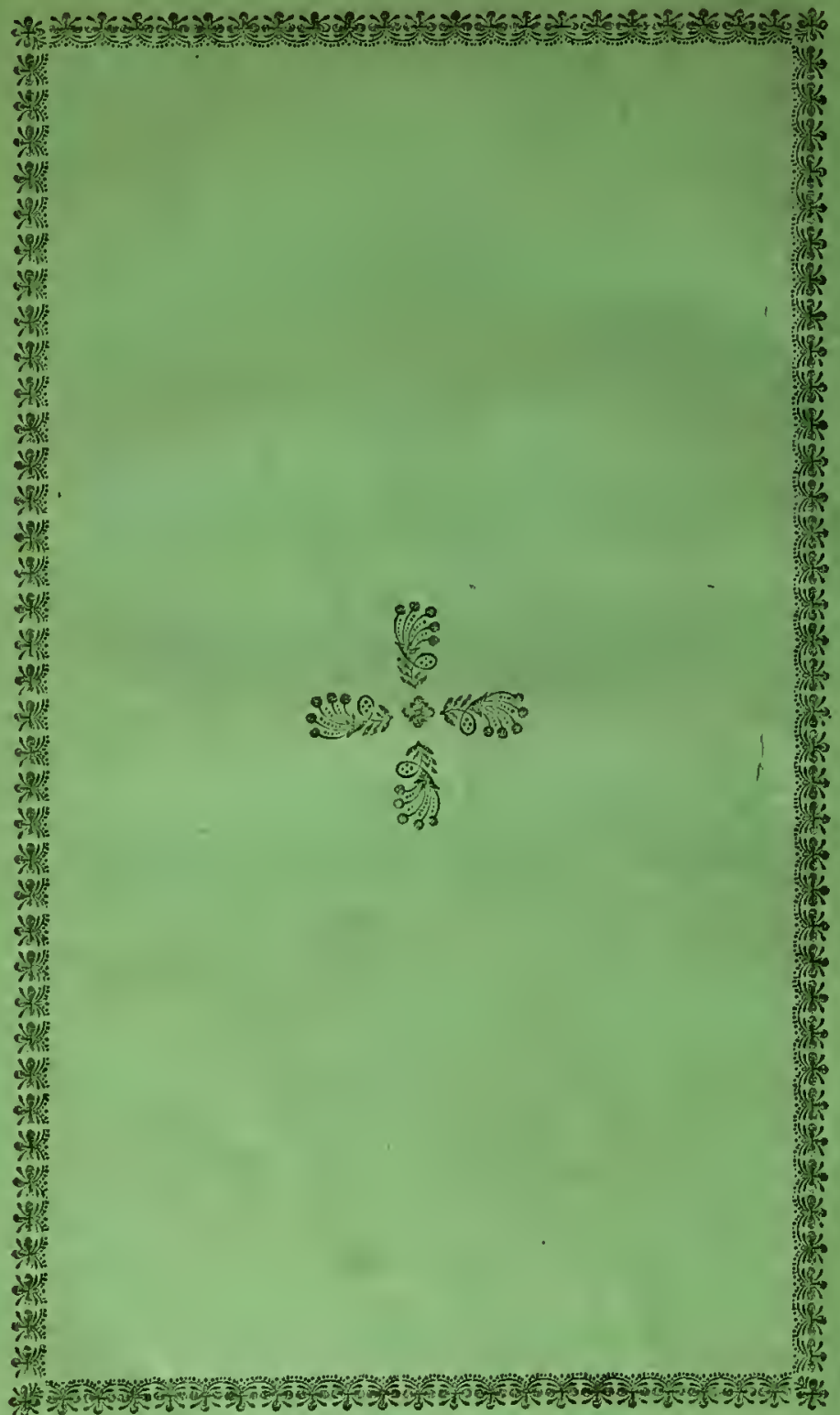

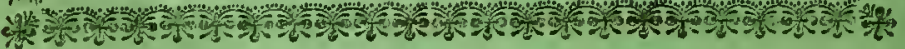

